\title{
Magnesium Alloys for Open-Pored Bioresorbable Implants
}

\author{
HANS JÜRGEN MAIER (®), ${ }^{1,4}$ STEFAN JULMI ${ }^{1}$ SABINE BEHRENS, ${ }^{1}$ \\ CHRISTIAN KLOSE, ${ }^{1}$ ANN-KATHRIN GARTZKE, ${ }^{2}$ PETER WRIGGERS, ${ }^{2}$ \\ ANJA-CHRISTINA WASELAU, ${ }^{3}$ and ANDREA MEYER-LINDENBERG ${ }^{3}$
}

\begin{abstract}
1.-Institut für Werkstoffkunde (Materials Science), Leibniz Universität Hannover, An der Universität 2, 30823 Garbsen, Germany. 2.-Institute of Continuum Mechanics, Leibniz Universität Hannover, Appelstraße 11, 30167 Hannover, Germany. 3.-Clinic for Small Animal Surgery and Reproduction, Centre of Clinical Veterinary Medicine, Faculty of Veterinary Medicine, Ludwig-Maximilians-Universität München, Veterinärstr. 13, 80539 Munich, Germany. 4.-e-mail: maier@iw.uni-hannover.de
\end{abstract}

If bone defects occur, the body's own healing mechanism can close them below a critical size; for larger defects, bone autografts are used. These are typically cut from the same person's hip in a second surgery. Consequently, the risk of complications, such as inflammations, rises. To avoid the risks resulting from the second surgery, absorbable, open-pored implants can be used. In the present study, the suitability of different magnesium alloys as absorbable porous bone substitute material has been investigated. Using the investment casting process with its design flexibility, the implant's structure can be adapted to the ideal pore geometry with respect to bone ingrowth behavior. Different magnesium alloys (Mg-La2, LAE442, and ZX61) were studied and rated in terms of their degradation rate, bone ingrowth behavior, biocompatibility, and resorbability of the individual alloying elements.

\section{INTRODUCTION}

Bone is continuously under load, which can cause bone defects such as fatigue cracks. Such defects can be dealt with by the body's own healing mechanism. However, there is a critical size beyond which bone defects can no longer be repaired by this mechanism. ${ }^{1}$ Thus, such critical size defects (CSDs) have to be treated with a bone implant. The state-ofthe-art treatment for CSD uses allogenic or autologous bone material. Autologous bone grafts have to be cut off from the patient's bone (mostly the hip) in a second surgery. However, such extra treatments can cause inflammation and pain. ${ }^{2}$ Allogenic bone transplants originate from other people and consequently can lead to infections or transmission of diseases. $^{3}$

These problems can be avoided by using synthetic bone grafts. Bone substitutes can be made of ceramics, metals, or polymers. Except for metals, the implant material can be either synthetic such as alumina $\left(\mathrm{Al}_{2} \mathrm{O}_{3}\right)$ and polylactic acid (PLA) or natural such as collagen or calcium phosphate $(\mathrm{CaP}) .{ }^{4-6}$ However, polymers and ceramics exhibit poor mechanical properties. Typically, the strength of polymers is too low for load-bearing implants. While the strength of ceramics is adequate in this respect, their fracture toughness is often not sufficient for cyclic loading ${ }^{7}$ or impact situations. In addition, the elastic modulus of ceramics is much higher than those of bone, which increases the likelihood of stress shielding effects. ${ }^{8}$

Conventional metal-based bone substitute materials are $\mathrm{Ti}$ alloys, Co-Cr alloys, and surgical steel. However, these metals have a high elastic modulus as compared with natural bone, which can also cause stress shielding. ${ }^{9}$ Magnesium $(\mathrm{Mg})$ alloys have a much lower elastic modulus. Thus, the stiffness of Mg-based implants can be more easily tailored to match that of bone, which reduces the risk of stress shielding. The strength of $\mathrm{Mg}$ alloys is lower than that of conventional bone substitute materials such as Ti or alumina, but is still sufficient for bone substitution. ${ }^{10}$ However, the corrosion resistance of $\mathrm{Mg}$ and its alloys is rather poor and thus must be increased substantially. The degradation behavior can be improved by alloying, adapting the microstructure, or application of coatings. ${ }^{11,12}$ In vivo studies by Angrisani et al. demonstrated a low in vivo degradation rate of dense scaffolds made 
of LAE442 alloy in a rabbit model. Part of the $\mathrm{Mg}$ scaffold was still present after 3.5 years. LAE442 also showed sufficient biocompatibility during the investigation period. ${ }^{13}$ Depending on the application in mind, the degradation time might have to be lower. It also turned out that the use of pure rareearth elements instead of a mixture of rare earths led to more reproducible results. Willbold et al. investigated the degradation rate of $\mathrm{Mg}-\mathrm{La} 2, \mathrm{Mg}$ $\mathrm{Nd} 2$, and Mg-Ce2 alloys. Mg-La2 showed a moderate corrosion rate in addition to good biocompatibility. ${ }^{14}$

Reducing the degradation rate of $\mathrm{Mg}$ alloys is one possibility; raising the bone ingrowth behavior is another one. The latter can be tailored via the pore size of open-pored scaffolds. The ideal pore size is reported to lie in the range of $150 \mu \mathrm{m}$ to $500 \mu \mathrm{m} .^{12,15}$ In earlier studies, a defined pore arrangement with the necessary pore sizes could be realized using an investment casting process. Furthermore, the resulting open-pored $\mathrm{Mg}$ scaffolds showed sufficient strength for the envisaged application. ${ }^{11,16}$

Apparently, an ideal scaffold would promote bone ingrowth behavior, provide sufficient mechanical strength, and degrade after a given time without leaving any critical elements within the body. The objective of the present study is to move one step ahead in this direction by studying three different Mg-based alloys under conditions relevant for the clinical application. To tailor the degradation behavior, three different coatings were included in the study. As detailed below, the new ZX61 is a promising candidate aluminum- and rare-earth-free alloy that can be cast to realize bioresorbable implants with adequate mechanical properties and tailored degradation behavior.

\section{EXPERIMENTAL PROCEDURES}

\section{Magnesium Alloys}

In the present study, the $\mathrm{Mg}$ alloys LAE442 (4 wt.\% Li, 4 wt.\% Al, 2 wt.\% rare earth mischmetal and balance $\mathrm{Mg}$ ), La2 (2 wt.\% $\mathrm{La}$ and balance $\mathrm{Mg}$ ), and ZX61 (6 wt.\% Zn, 1 wt.\% Ca, and balance Mg) were used. LAE442 was produced by adding lithium to commercial AE42 alloy. Given the high reactivity of magnesium-lithium alloys, the alloy was cast in an argon atmosphere at 1.2 bar and a temperature of $750^{\circ} \mathrm{C}$ followed by a 30 -min continuous stirring process; for details see Seitz et al. ${ }^{17}$ Due to the high melting point of lanthanum (La), the process suggested by Weizbauer et al. ${ }^{18}$ was employed to manufacture Mg-La2. In this approach, Mg-La40 foundry alloy and pure magnesium were melted at $750^{\circ} \mathrm{C}$ then stirred for $30 \mathrm{~min}$ under argon atmosphere to obtain the final binary Mg-La2 alloy.

ZX61 alloy was cast by gravity die casting using high-purity $\mathrm{Mg}$, pure $\mathrm{Zn}$, and $\mathrm{Mg}$-Ca30 master alloy. In this case, the magnesium was heated in a steel crucible to $750^{\circ} \mathrm{C}$ under a protective gas flow consisting of nitrogen with 0.3 vol.\% sulfur hexafluoride $\left(\mathrm{SF}_{6}\right)$. The $\mathrm{Mg}-\mathrm{Ca} 30$ master alloy was added to the $\mathrm{Mg}$ melt, which was then stirred for $30 \mathrm{~min}$. Thereafter, $\mathrm{Zn}$ was introduced into the melt, followed by another $20 \mathrm{~min}$ of stirring. Finally, the melt was cast into a steel mold.

Note that the alloys studied by Seitz et al. ${ }^{17}$ and Weizbauer et al. ${ }^{18}$ were designed with different applications in mind. Thus, these alloys were extruded after casting, whereas the scaffolds in the present study were cast directly. Consequently, the microstructures and properties are different from the extruded alloys despite their similar chemical composition. The actual microstructures of all three different alloys studied are shown in Fig. 1. Obviously, the grain size is larger in case of ZX61, which can be attributed to differences in cooling rates upon casting. As addressed below, ZX61 still exhibits mechanical properties that are sufficient for the envisaged application.

\section{Sample Preparation}

The sample manufacturing process comprised three stages. The initial step included the design of the geometry of the scaffold and the analysis of its load-bearing capacity by finite element analysis. Next, additive manufacturing (AM) was used to obtain wax casting models. For AM, the threedimensional (3D) computer-aided design (CAD) file was cut into layers of $12.7 \mu \mathrm{m}$ using ModelWorks software (Solidscape, Inc., Merrimack, USA). The casting model was then manufactured layer by layer using the fused deposition modeling method with a T612BT2 (Solidscape, Inc., Merrimack, USA) from two types of waxes, viz. Indura Cast (Solidscape, Inc., Merrimack, USA) for the model and Indura Fill (Solidscape, Inc., Merrimack, USA) for the support structure. Both are specifically made for investment casting. The support structure could be solved out afterwards using a petroleum bath at $45^{\circ} \mathrm{C}$ (VELIND Aerosol GmbH, Schwedt, Germany). Next, the casting models were welded onto a wax rod to produce a casting tree. The casting tree was then embedded under vacuum in Gilcast AM (BK Giulini GmbH, Ludwigshafen, Germany), a mold material specifically developed for magnesium investment casting. During the curing process of the mold material, the wax was burned out and the mold was kept at $300^{\circ} \mathrm{C}$ and $350^{\circ} \mathrm{C}$, when casting the scaffolds with LAE442 and Mg-La2, respectively. The mold material was solved out afterwards using a special gypsum-dissolving agent (SRL Dental GmbH, Ludwigshafen, Germany). It turned out that the scaffold design used in previous investigations for in vivo studies with LAE442 ${ }^{11}$ could be cast in $\mathrm{Mg}-\mathrm{La} 2$ as well, and the present paper reports the data obtained with this design.

In addition, dense samples of LAE442 and ZX61 alloys were cast in a steel mold preheated to $300^{\circ} \mathrm{C}$. The actual casting process was conducted in a benchtop MC50 casting furnace (Indutherm $\mathrm{GmbH}$, 



Fig. 1. Optical micrographs of as-cast (a) Mg-La2, (b) LAE442, and (c) ZX61 alloys, revealing the differences in grain size

Walzbachtal-Wössingen, Germany). For casting of Mg-La2 and ZX61, the furnace chamber was evacuated, filled with argon, and evacuated a second time, while preheating the crucible to $350^{\circ} \mathrm{C}$. Finally, the $\mathrm{Mg}$ alloy was heated up to $750^{\circ} \mathrm{C}$ for casting. The casting process had to be slightly modified for LAE442. After the second evacuation of the furnace chamber, it was filled again with argon for the casting and a casting temperature of $740^{\circ} \mathrm{C}$ was used. The dense samples were machined to a cylindrical geometry with diameter of $4 \mathrm{~mm}$ and length of $5 \mathrm{~mm}$. The open-pored scaffolds already featured a diameter of $4 \mathrm{~mm}$, and thus had only to be turned to length of $5 \mathrm{~mm}$.

\section{In Vitro Degradation}

The in vitro degradation behavior was studied using simulated body fluid ( $\mathrm{r}$-SBF) after Oyane et al. ${ }^{19}$ Each sample was deposited in a vessel with $50 \mathrm{ml}$ SBF. To ensure homogeneous corrosion attack, the vessels were placed on an orbital shaker (GFS 3017, Gesellschaft für Labortechnik $\mathrm{mbH}$, Burgwedel, Germany). For each set of parameters (alloy, period of time, coating, and shape), three samples were employed. After a given period of time, the samples were removed from the SBF, flushed with ethanol, and treated in chromic acid with chromium(VI) oxide (Merck KGaA, Darmstadt, Germany), silver nitrate, und barium nitrate (both 99\% purity; Carl Roth GmbH \& Co. KG, Karlsruhe, Germany) according to standard DIN ISO 8407:144 to remove the adherent corrosion product. Volume and mass were measured for the initial samples and the samples before and after the acid treatment. The mass was measured by weighing, and the volume was determined using the Archimedes principle. Three-dimensional (3D) tomographic images to assess the interior corrosion attack were obtained by high-resolution X-ray microscopy (XRM) using an Xradia 520 Versa (Carl Zeiss AG, Oberkochen, Germany) with a spatial resolution of $3.5 \mu \mathrm{m}$ at an acceleration voltage of $40 \mathrm{kV}$, energy of $3 \mathrm{~W}$, and exposure time of $2 \mathrm{~s}$.

In the in vitro corrosion tests, the influence of different coatings on the degradation behavior was also analyzed. For all coated samples, a magnesium fluoride $\left(\mathrm{MgF}_{2}\right)$ layer was formed by first boiling the samples for $2 \mathrm{~h}$ in $5 \mathrm{M}$ sodium hydroxide solution, which resulted in the formation of a magnesium hydroxide layer. This layer was then converted to $\mathrm{MgF}_{2}$ by treatment in $40 \%$ hydrofluoric acid for $96 \mathrm{~h}$. Part of the samples received an additional calcium phosphate (CaP) or polylactide acid (PLA) top layer. Details on the processing steps needed to form the top layers are given in Ref. 11. These coatings are designated hereinafter as $\mathrm{MgF}_{2}$, $\mathrm{MgF}_{2}+\mathrm{CaP}$, and $\mathrm{MgF}_{2}+$ PLA.

\section{In Vivo Degradation}

For analysis of the in vivo degradation behavior, only $\mathrm{MgF}_{2}$-coated scaffolds were used in the present study. The in vivo studies were performed using the procedure described by Kleer et al. with rabbits. ${ }^{20}$ After 6 weeks, 12 weeks, 24 weeks, and 36 weeks, the scaffolds were explanted and the bone implant compounds were fixed in $4 \%$ paraformaldehyde for 10 days to 14 days. For detailed scanning electron microscopy (SEM) analysis, they were dehydrated in an ascending series of alcohol, defatted in xylene, and infiltrated and embedded in Technovit 9100 (Kulzer GmbH, Wehrheim, Germany). Thick sections of about $150 \mu \mathrm{m}$ were cut using a diamond band saw (Cut Grinder, Walter Messner GmbH, Oststeinbek, Germany) then ground (Lap-Grinder, Walter Messner GmbH, Oststeinbek, Germany) to final thickness of $50 \mu \mathrm{m}$ to $60 \mu \mathrm{m}$ and polished. Images of the slices were then recorded by SEM (SUPRA 55 VP; Carl Zeiss AG, Oberkochen, Germany) with $\mathrm{x}$-ray spectroscopy (EDX) analysis at an acceleration voltage of $15 \mathrm{kV}$.

\section{RESULTS}

\section{In Vitro Degradation of Mg Scaffolds}

Because of their porous structure, the total surface of the investigated scaffolds was very large. Thus, the mass loss was expected to be higher than for dense samples. However, the degradation rate of the Mg-La2 scaffolds in SBF far surpassed expectations. Figure 2 shows 3D images of the scaffolds before and after the corrosion tests. If no coating was applied, the scaffolds were fully degraded after 

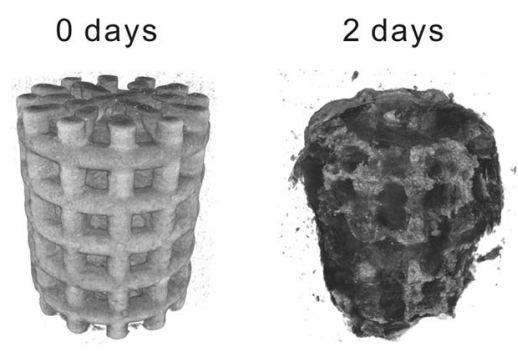

$\overline{1 \mathrm{~mm}}$

(a) uncoated
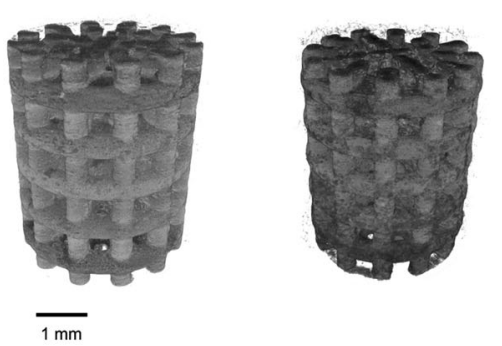

(b) $\mathrm{MgF}_{2}$
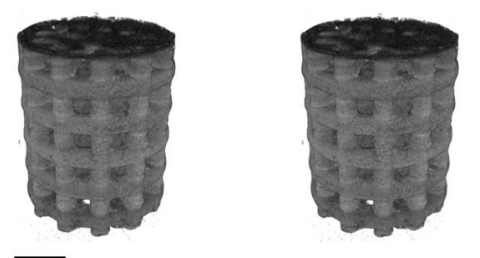

(c) $\mathrm{MgF}_{2}+\mathrm{CaP}$
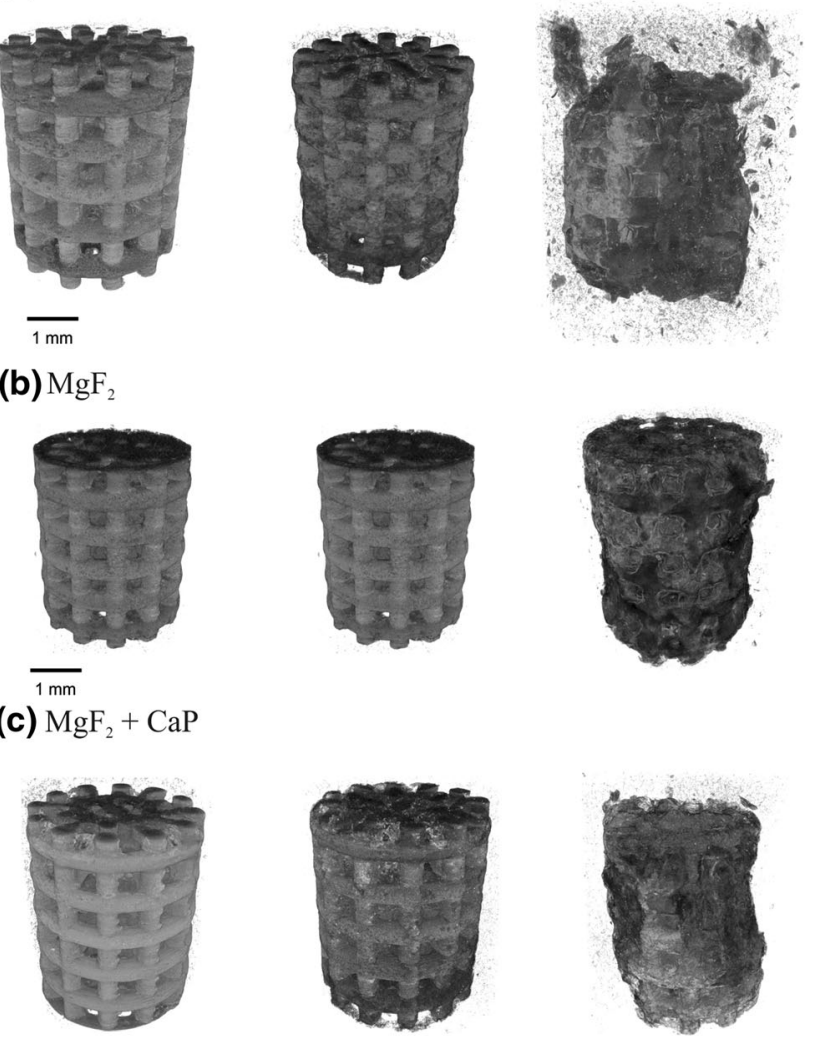

d) $\mathrm{MgF}_{2}+\mathrm{PLA}$

Fig. 2. Three-dimensional tomographic images of the Mg-La2 scaffolds in (a) uncoated and (b-d) coated conditions: virgin sample (left column), after 2 days (middle column) and after 5 days (right column) in SBF; pore size is $0.5 \mathrm{~mm}$ for all samples

5 days (Fig. 2a). However, the use of different coatings substantially diminished the degradation rate. After 5 days, the scaffold's structure was still preserved (Fig. 2b). However, the scaffolds were completely degraded after 8 days, which is clearly too short. Moreover, the two-dimensional (2D) slices in Fig. 3 clearly demonstrate that the corrosion attack was inhomogeneous, with slices showing indications of pitting corrosion. At a few spots, the corrosion attack went deep into the individual elements. Moreover, even after 5 days of corrosion, it was still not distributed over the entire surface (Fig. 3b). Note that the cross sections do not show the same plane; Thus, at first glance, Fig. 3b might look less corroded as it shows more of the metal. However, the gray-appearing degradation layer is clearly much more prominent after the longer exposure (Fig. 3b) than after 2 days (Fig. 3a). Interestingly, the corrosion was most pronounced in the central part of the scaffold structure, which indicates a local change in the composition of the electrolyte due to limited exchange with the SBF surrounding the scaffold.

A strong effect of grain size on the corrosion rate has been reported for LAE442, ${ }^{11}$ thus the bottom and top parts of the scaffold degraded differently due to the different cooling rate and grain size there. By contrast, the Mg-La2 scaffold showed no pronounced difference in corrosion attack between the top and bottom (Fig. 2). Only the $\mathrm{MgF}_{2}+\mathrm{CaP}-$ coated scaffold showed slightly faster degradation at the gating part.

Although Mg-La2 is used here to illustrate the extremely rapid degradation of this alloy, the general trend seen in Fig. 2, i.e., a reduction in the degradation rate upon applying the different coatings, was also observed for the other alloys studied. However, there were substantial differences between the alloys in terms of their degradation rate. Comparing the degradation behavior with previous results for LAE442, Mg-La2 degraded much faster (Fig. 4). Even the uncoated LAE442 scaffolds retained $35 \%$ of their initial mass after 12 weeks. For both alloys, the combined $\mathrm{MgF}_{2}+$ PLA was the most effective coating for reducing the degradation rate. As demonstrated in an earlier study, ${ }^{11}$ the mechanical properties of both Mg-La2 and LAE442 are sufficient for the typical scaffold application considered here. However, only coated LAE442 exhibited a degradation rate slow enough for the current context.

\section{In Vivo Degradation}

The in vitro corrosion results can provide an indication of the in vivo behavior for a comparison between the different alloys. However, it is also necessary to determine their actual in vivo behavior. Thus, both LAE442 and Mg-La2 were tested in vivo, although the in vitro results indicated that Mg-La2 might degrade too rapidly. Clinical evaluation of LAE442 and Mg-La2 scaffolds has already been reported by Kleer et al. ${ }^{20}$ In that study, $\mathrm{MgF}_{2^{-}}$ coated scaffolds were inserted into the cancellous part of the greater trochanter of both femurs in rabbits and evaluated over a period of 36 weeks. Regular clinical, radiological, and in vivo microcomputed tomography $(\mu \mathrm{CT})$ examinations were employed, revealing no adverse clinical reactions to any of the scaffolds. In the following, the corresponding structural and microstructural evolution is analyzed. Figure 5 shows electron backscatter images of one cross section of each scaffold. The metallic parts have a higher average atomic number density than the reaction products and thus appear 

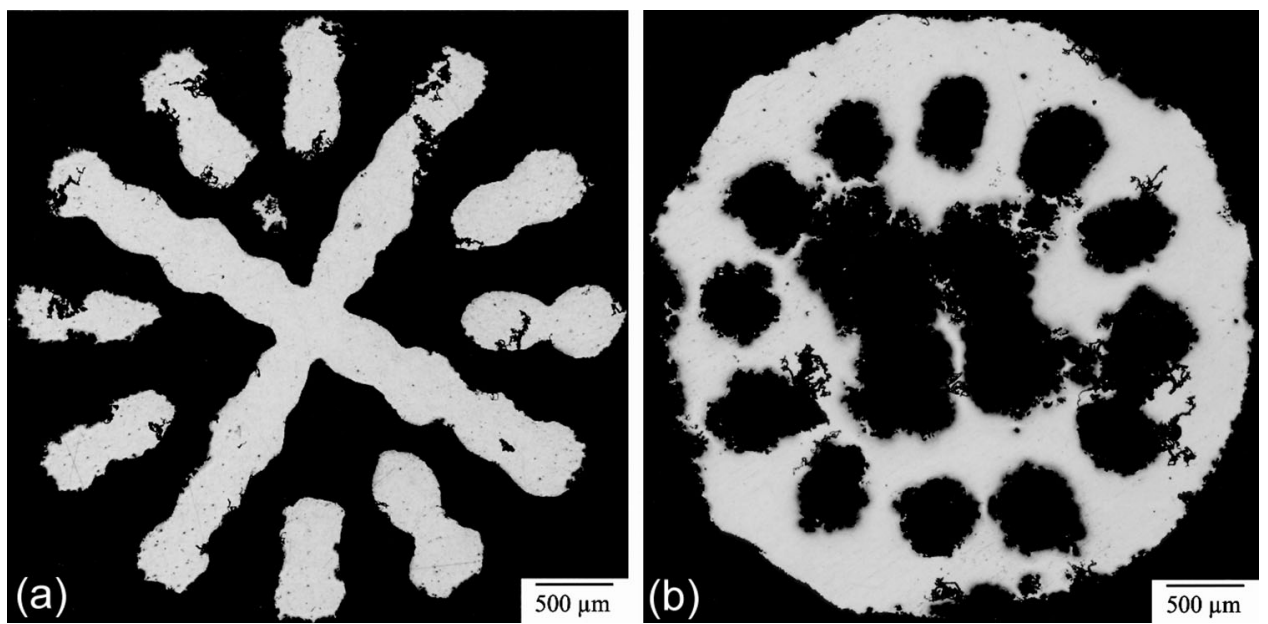

Fig. 3. Optical cross sections of Mg-La2 scaffolds with $\mathrm{MgF}_{2}+\mathrm{PLA}$ coating after (a) 2 days and (b) 5 days in SBF; see main text for details

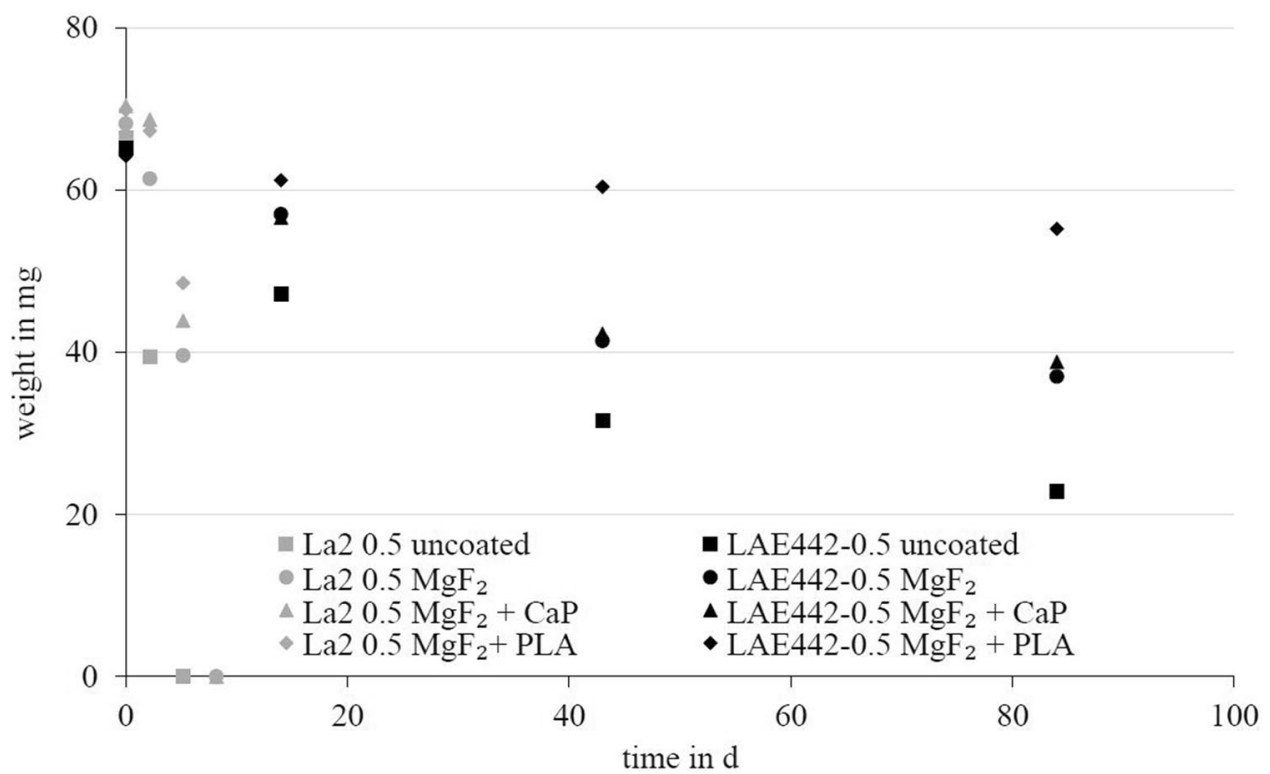

Fig. 4. Weight of Mg-La2 and LAE442 scaffolds after different degradation times; symbols represent the average data from three specimens with maximum difference in weight being less than $4 \mathrm{mg}$ for each set

brighter. Obviously, the relation between the degradation rates of LAE442 and $\mathrm{Mg}-\mathrm{La} 2$ is similar to the trend observed in the in vitro tests. However, the in vivo corrosion rate is much slower than that observed in vitro. Indeed, only about $50 \%$ of the cross section of $\mathrm{Mg}-\mathrm{La} 2$ was degraded after 6 weeks (Fig. 5e), while in case of LAE442, nearly no corrosion attack of the scaffolds was noted after the same amount of time (Fig. 5a). Another substantial difference between LAE442 and Mg-La2 is the change in the appearance of the scaffold structure upon degradation. While the overall structure of LAE442 could still be recognized after 36 weeks, the structure of Mg-La2 was already fully disrupted in some parts after 6 weeks. Clearly, there is a pronounced difference between the in vitro and in vivo behavior, but Mg-La2 would still degrade too rapidly for most applications.

With respect to the envisaged application, the composition of the degradation layer and its absorption rate by the body are also of interest. Depending on the time of exposure in the rabbit, different degradation phases could be noted. Representative compositions at the locations indicated in Fig. 5 on the two explanted scaffolds are presented in Table I. The four key areas are the initial noncorroded alloy, the degradation layer, the material deposited on the surface of the implanted scaffolds, and the residues of the degraded scaffolds. The latter was only detected for the Mg-La2 scaffolds. The C- and Ocontent were excluded from the EDX analyses, because the concentration of these elements is 

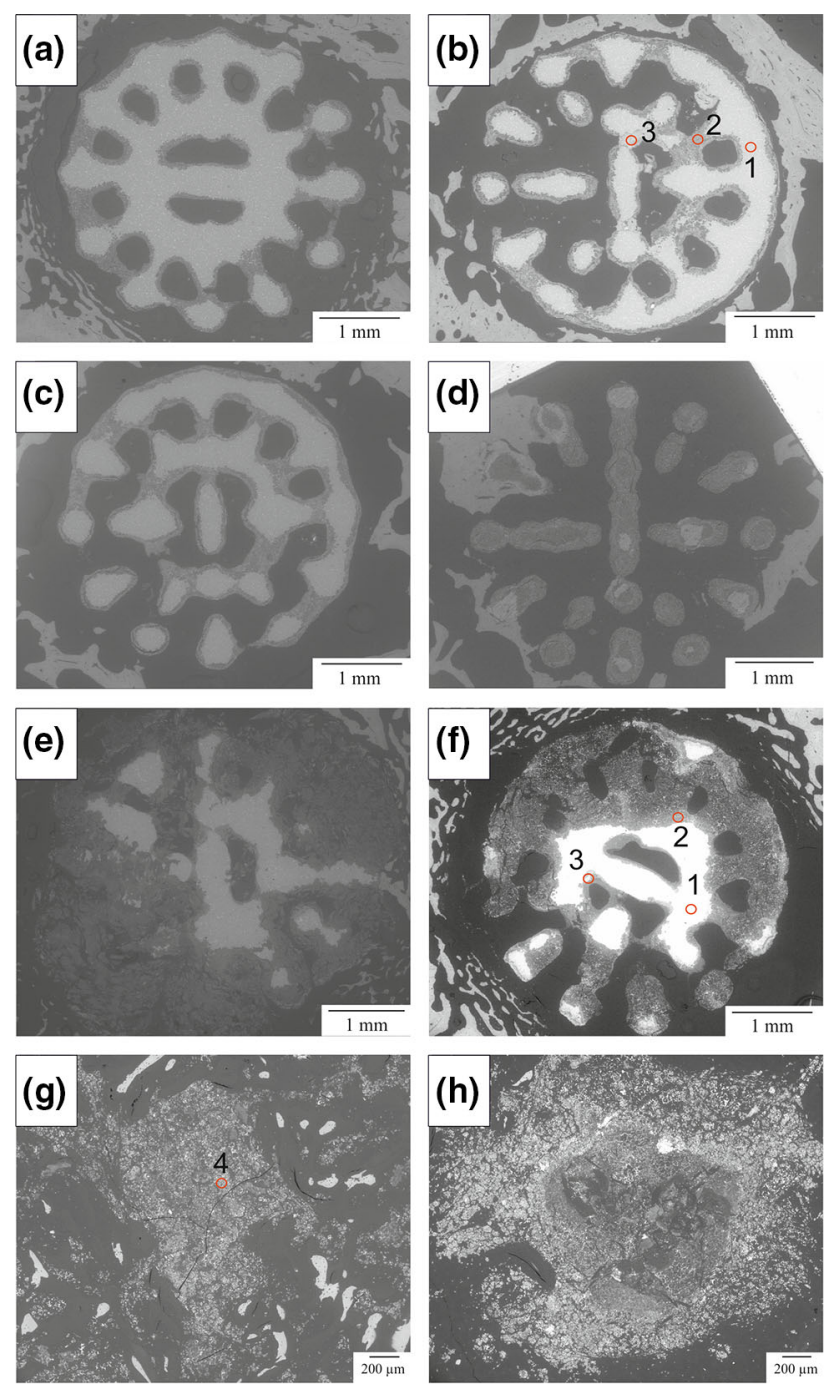

Fig. 5. SEM images of explanted scaffolds using electron backscatter contrast: (a-d) LAE442 and (e-h) Mg-La2 after (a, e) 6 weeks, (b, f) 12 weeks, (c, g) 24 weeks, and (d, h) 36 weeks; the positions marked in (b), (f), and (g) indicate the location of the EDX analysis presented in Table I

difficult to quantify by EDX. The composition of the noncorroded alloys was close to the nominal value expected for LAE442 and Mg-La2. Only a few additional elements were present, at low contents, which can be attributed to preparation artifacts. Note that EDX probes a volume a few microns in size, thus material hidden underneath the surface will contribute to the signal, which can explain why the composition varied noticeably within the degradation layer. Still, the $\mathrm{Ca}$ and phosphorus (P) content in the degradation layer (position 2) and in the material deposited on the LAE442 scaffold (position 3) were high in each case for all samples. In fact, the average EDX data obtained at these locations did not change significantly between scaffolds explanted after different times; i.e., only the amount of the degraded part of the scaffold changed, but not its average chemical composition. However, the results also demonstrated that $\mathrm{Mg}$ dissolved while $\mathrm{Al}$ remained within the degradation layer for LAE442 (Table I, position 2). This enrichment in the Al content may be critical in terms of long-term biocompatibility. In contrast, the amount of $\mathrm{Ca}$ and $\mathrm{P}$ in the degradation layer and the deposited material was drastically lower for the Mg-La2 than LAE442 scaffolds (Table I, positions 2 and 3).

In the material that remained after full degradation of the Mg-La2 scaffolds (Table I, position 4), the La content increased tremendously, demonstrating that this rare earth is hardly absorbed by the body. The increased iron content detected by EDX analysis is a typical impurity in commercially available La but is not critical for the body. Although both types of scaffolds did not show any clinical complications, the long-term effects of the remaining $\mathrm{Al}$ and rare earths are still unknown. Consequently, new alloys with elements such as $\mathrm{Zn}$ and $\mathrm{Ca}$, which exist as trace elements in the body and are more soluble, appear promising. ${ }^{21}$ Corrosion resistance is known to increase when alloying with $\mathrm{Zn}$ or adding 0.8 wt.\% Ca. ${ }^{22}$ Thus, in the present study, the ZX61 alloy with a $\mathrm{Zn}$ content slightly below the solubility limit of $\mathrm{Zn}$ in $\mathrm{Mg}$ and a Ca content of 1 wt.\% was tested as well. In addition, this new alloy should have a degradation behavior that is tailored slightly better to the application than LAE442.

\section{In Vitro Degradation of ZX61}

The objective of the alloy development was to realize an alloy with corrosion properties similar to LAE442 during the initial phase but that degrades faster after reaching the end of the functional phase, when the bone can bear the load on its own. To evaluate the corrosion behavior of the new alloy ZX61, in vitro tests were conducted. As Mg-La2 degrades too fast, only LAE442 is used as the reference in the following.

If the amount of magnesium oxides and hydroxides is minimal in the initial phase, then the $\mathrm{Mg}$ concentration in the SBF reflects the corrosion rate. The $\mathrm{Mg}$ content measured in the SBF during the first 2 days is shown in Fig. 6. Within the experimental scatter, the $\mathrm{Mg}$ concentration in the SBF is similar for both alloys up to $6 \mathrm{~h}$. After this initial, nearly linear phase, the corrosion rate slows down between $6 \mathrm{~h}$ and $24 \mathrm{~h}$ for both alloys. For LAE442 alloy, it even seems to reach a saturation stage after $48 \mathrm{~h}$. By contrast, the Mg concentration of the SBF for the ZX61 samples was still increasing, albeit only weakly. This indicates that ZX61 might form a less protective degradation layer, in turn resulting in the intended faster degradation rate upon longterm use.

However, as soon as the $\mathrm{Mg}^{2+}$ ion concentration approaches saturation, its concentration is no longer a good indicator of the corrosion rate. Thus, a different method was used to obtain data on the long-term degradation behavior. In these tests, the 
Table I. Composition of Mg-La2 and LAE442 scaffolds after explantation (6 weeks) for the uncorroded part (position 1), degradation layer (position 2), material deposited on the surface of the scaffolds (position 3), and residues after full degradation (only observed for Mg-La2, after 36 weeks, positions 4); Positions 1 to 4 are marked in Fig. 5b, $f$, and $g$

Proportion in wt.\%

\begin{tabular}{|c|c|c|c|c|c|c|c|}
\hline \multirow[b]{2}{*}{ Element } & \multicolumn{3}{|c|}{ LAE442 } & \multicolumn{4}{|c|}{ Mg-La2 } \\
\hline & Pos. 1 & Pos. 2 & Pos.3 & Pos. 1 & Pos. 2 & Pos. 3 & Pos. 4 \\
\hline $\mathrm{Mg}$ & 92.3 & 24.8 & 28.7 & 98.8 & 92.5 & 63.4 & 2.3 \\
\hline $\mathrm{Al}$ & 3.0 & 12.2 & 1.0 & & & & 3.1 \\
\hline $\mathrm{La}$ & 0.2 & 0.5 & & 1.1 & 1.1 & 0.5 & 61.7 \\
\hline $\mathrm{Ce}$ & 0.7 & 2.5 & & & & & \\
\hline $\mathrm{F}$ & & & 0.9 & & 0.6 & 22.8 & \\
\hline $\mathrm{Na}$ & & 1.2 & 3.0 & & & 2.0 & \\
\hline $\mathrm{Si}$ & 0.3 & 0.8 & 0.2 & & & & \\
\hline $\mathrm{P}$ & 1.1 & 23.8 & 19.5 & & & 5.3 & 19.6 \\
\hline S & 0.7 & 0.3 & & & & & 2.0 \\
\hline $\mathrm{Cl}$ & & & & & 5.1 & & \\
\hline K & & & 0.1 & & & 0.3 & \\
\hline $\mathrm{Ca}$ & 1.7 & 33.9 & 46.6 & 0.6 & 0.7 & 5.7 & 9.8 \\
\hline $\mathrm{Fe}$ & & & & & & & 1.5 \\
\hline
\end{tabular}

samples were removed after 2 weeks of degradation and the corrosion rate was calculated from the weight and volume loss of the samples. The corresponding data are shown in Figs. 7 and 8 and Table II. After 2 weeks, the volume of the LAE442 samples was still $91.5 \%$ of the initial value while the weight was $88.5 \%$ of the initial value, whereas the ZX61 samples had lost $45 \%$ of their volume and $46 \%$ of their weight. However, the degradation layer of the LAE442 samples represented a higher weight fraction (16.3\%) than observed for the ZX61 alloy $(12.8 \%)$. This indicates that the corrosion layer of LAE442 was more stable. However, for bone implants, the degradation rate should be moderate and the degradation layer should be resorbed by the body in the long term. Thus, the ZX61 alloy with its degradation rate similar to LAE442 in the initial phase and subsequent increase appears to be advantageous.

In addition to this quantitative analysis of the in vitro corrosion behavior, tomographic images of the samples were obtained (Fig. 9). These data are in good agreement with the more macroscopic volume measurement made on the corroded samples. The initial geometries of both samples were identical. Figure 9 demonstrates that, after 2 weeks, the differences in degradation behavior were quite obvious. Not only was the remaining cross section of the LAE442 much larger than that of the ZX61 alloy, but the corrosion attack was also much more homogeneous in the former. The cross section of the ZX61 alloy demonstrated the typical signature of localized pitting corrosion. Comparing the corrosion layers, the LAE442 samples featured a homogeneous corrosion layer (the darker outer layer in Fig. 9). Obviously, this dense layer is an effective barrier for further corrosion attack and the reason for the slow degradation of this alloy. ZX61, on the contrary, formed nearly no protective corrosion layer. Indeed, the degradation layer was even smaller then expected based on the quantitative analysis of the in vitro tests. The discrepancy between the thickness of the degradation layer on ZX61 seen in Fig. 9 and the fraction of the layer calculated based on the mass and volume of the samples can be partly attributed to the low contrast of this layer in the tomography images and an attack of the substrate itself upon removing the degradation layer using chromic acid.

\section{DISCUSSION}

In the present study, the mechanical behavior of the three different Mg-based alloys studied was not addressed in detail, although the alloys exhibit substantially different strength. Specifically, previous results have already shown that the maximum tolerable load within the elastic regime $(858 \mathrm{~N})$ of Mg-La2 implants is significantly lower than that of LAE442 implants (1608 N). ${ }^{11,16}$ However, this lower value is still sufficient for the implant's location considered within the rabbit's tibia, as the bone is subjected to only a maximum load of $60 \mathrm{~N}$ there. ${ }^{23,24}$

However, with respect to the degradation behavior, the difference between the alloys is much more crucial. Whereas parts of the implants with LAE442 were still not degraded after 12 weeks, the Mg-La2 implants are fully degraded after 5 days. ${ }^{11} \mathrm{~A}$ common method to reduce the degradation rate of $\mathrm{Mg}$ implants is to coat the surface. An established 


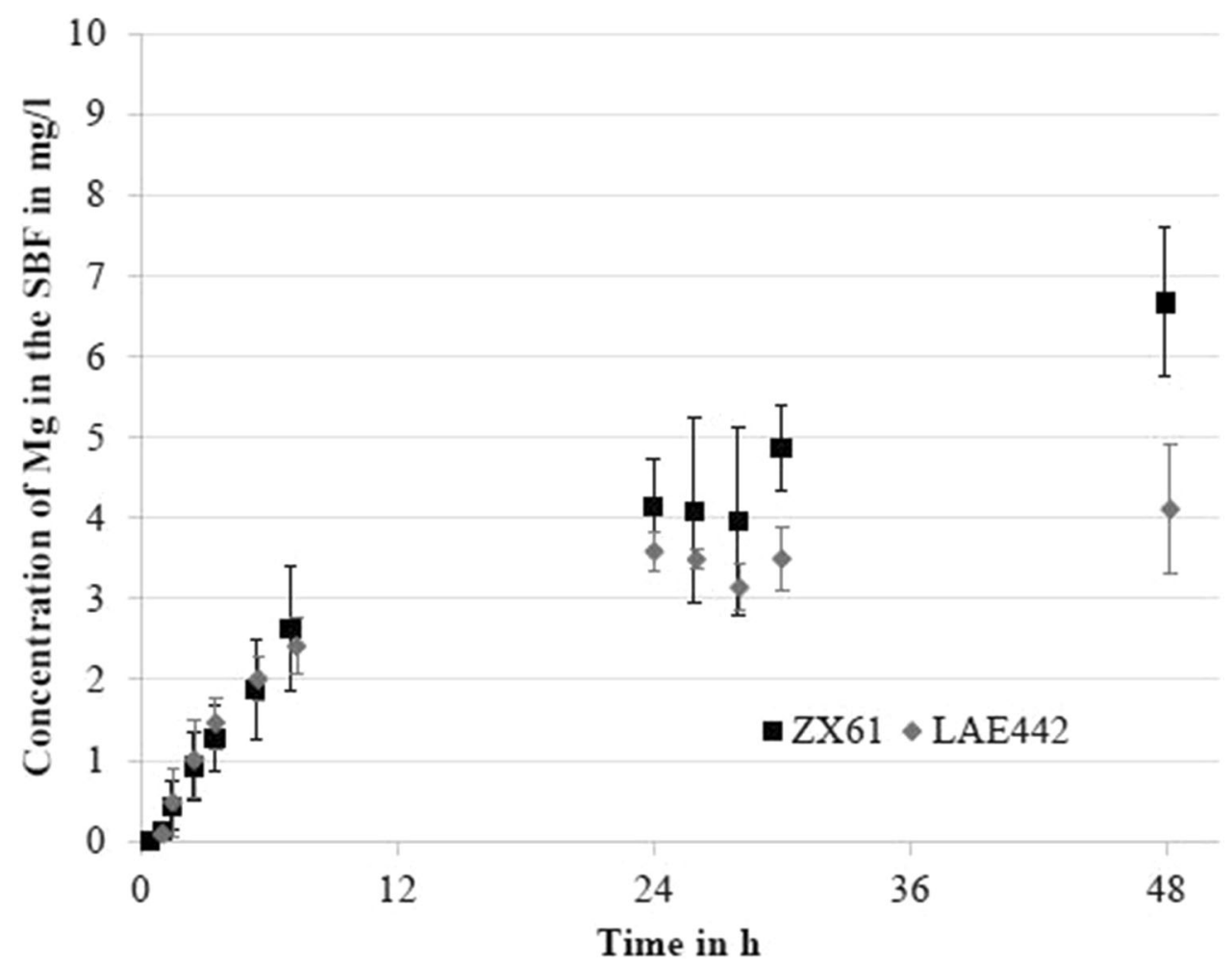

Fig. 6. Mg concentration in SBF for LAE442 and ZX61 alloys during the first $48 \mathrm{~h}$

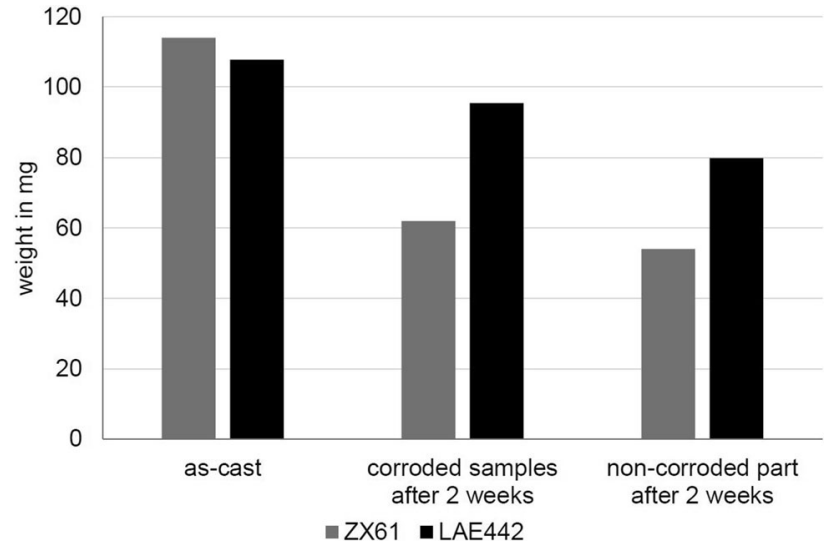

Fig. 7. Weight of ZX61 and LAE442 samples in as-cast condition, after in vitro corrosion, and after removing the corrosion layer

coating material for $\mathrm{Mg}$ implants is $\mathrm{MgF}_{2}$. Chiu et al. demonstrated a lower corrosion potential and current density of $\mathrm{MgF}_{2}$-coated pure $\mathrm{Mg}$. In electrochemical measurements, the corrosion attack of pure $\mathrm{Mg}$ after 2 days in Hank's solution was clearly visible, whereas $\mathrm{MgF}_{2}$-coated samples showed no signs of corrosion attack. ${ }^{25}$ Similarly, a reduced corrosion rate was demonstrated by Wolters et al. in investigations of the in vitro corrosion of LAE442 samples and by Thomann et al. using Mg-Ca0.8 alloy. ${ }^{26,27}$ By using $\mathrm{MgF}_{2}$ as a coating material, the weight loss of LAE442 as well as Mg-La2 could be reduced significantly.

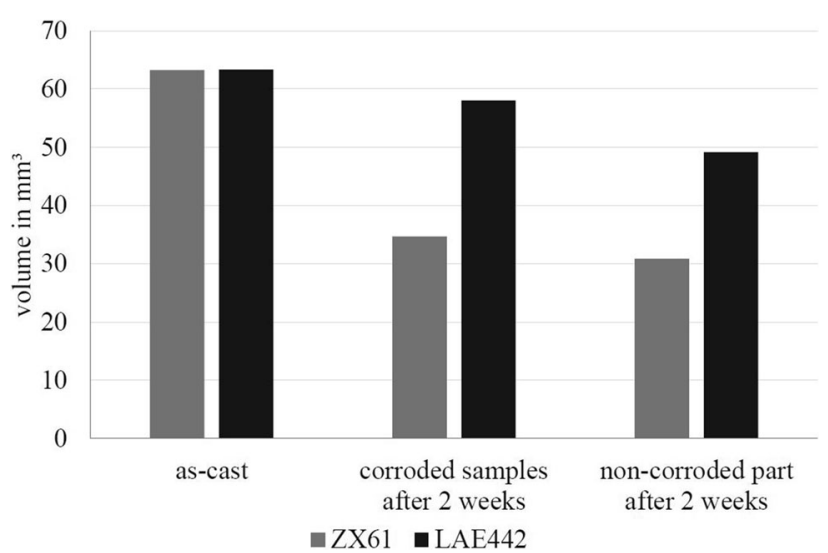

Fig. 8. Volume of ZX61 and LAE442 samples in as-cast condition, after in vitro corrosion, and after removing the corrosion layer

In the present study, additional coatings $(\mathrm{CaP}$ and PLA) were also employed on top of $\mathrm{MgF}_{2}$. This combination of coatings further decreased the weight loss. Zeng et al. investigated the in vitro corrosion of $\mathrm{Mg}-1.2 \mathrm{Li}-1.12 \mathrm{Ca}-1.0 \mathrm{Y}$ alloy with a micro-arc oxidation (MAO) and an MAO + PLA coating. When using only an MAO coating, the increase in $\mathrm{pH}$ value was already reduced over a period of $80 \mathrm{~h}$. The additional PLA coating kept the $\mathrm{pH}$ value at an even lower level. ${ }^{28} \mathrm{~A}$ different combination with hydroxyapatite (HA)-doped PLA was examined by Abdal-hay et al. for AZ31 alloy. The corrosion resistance of the doped and undoped coatings was similar, but the objective of adding HA 
Table II. Volume and mass of ZX61 and LAE442 samples before and after in vitro corrosion experiments

\begin{tabular}{|c|c|c|c|c|}
\hline \multirow[b]{2}{*}{ Condition } & \multicolumn{2}{|c|}{ Weight (mg) } & \multicolumn{2}{|c|}{ Volume $\left(\mathbf{m m}^{\mathbf{3}}\right)$} \\
\hline & ZX61 & LAE442 & ZX61 & LAE442 \\
\hline As-cast & 114 & 107.8 & 63.2 & 63.3 \\
\hline Corroded samples after 2 weeks & 61.9 & 95.5 & 34.6 & 58 \\
\hline Non-corroded part after 2 weeks & 54 & 79.9 & 30.8 & 49.2 \\
\hline
\end{tabular}
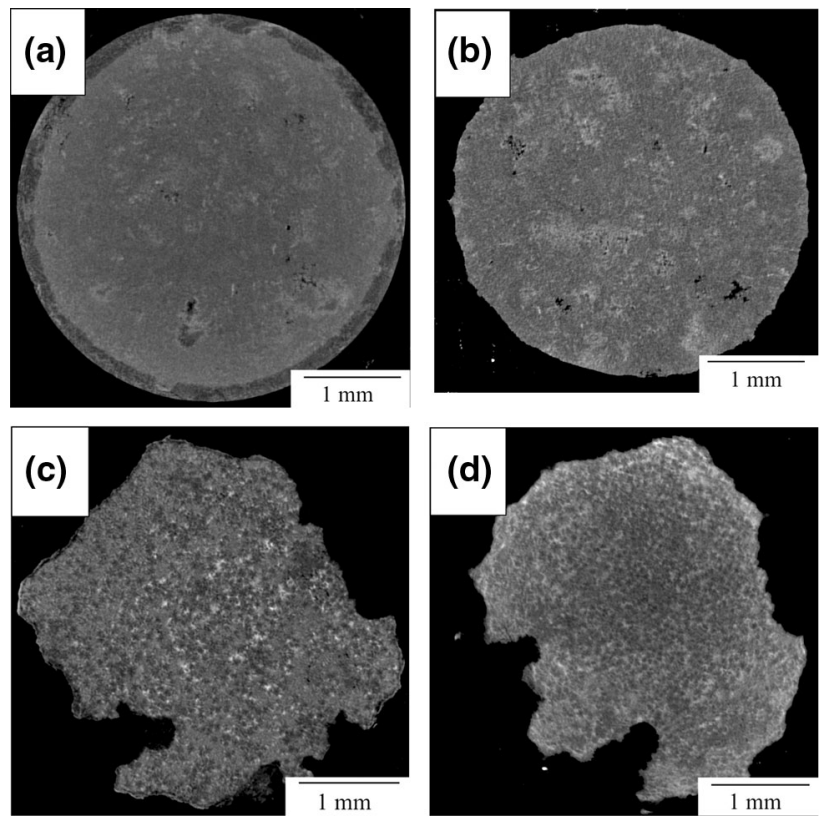

Fig. 9. Tomographic slices after 2 weeks in SBF of $(a, b)$ LAE442 and $(c, d) Z X 61$ with the corrosion layer $(a, c)$ present and $(b, d)$ ZX61 removed

was to enhance the bioactivity. ${ }^{29}$ Although PLA has high biocompatibility and can be used in drug delivery systems as well, calcium phosphate further enhances the activity of osteoblasts and the bone growth. ${ }^{30,31}$ Only considering the degradation rate, the additional PLA coating is the more effective variant as compared with the calcium phosphate coating. Tomographic images of LAE442 implants with the same coatings as used in the present study showed another benefit of the PLA coating: The effect of the grain size on the corrosion rate could be successfully suppressed. ${ }^{11}$

The present results demonstrate that in vivo the implants degrade much more slowly, but the relative corrosion rate between the alloys remains similar as in the in vitro experiments. Still, in vitro measurements cannot replace in vivo investigations. For instance, Mg-La2 alloy not only degraded faster, but the implant's structure was also fully lost rapidly. In contrast, the structure of the implants made of LAE442 alloy was still present after 36 weeks and thus will still have some load- bearing capacity, whereas such effects cannot be reliably predicted based on in vitro experiments alone.

In addition to the alloying elements, $\mathrm{Ca}$ and $\mathrm{P}$ were detected in significant amounts. Thus, it is very likely that calcium phosphate was formed. In clinical studies, no complications or negative effects such as lameness or pain were observed when using LAE442 and Mg-La2 scaffolds. ${ }^{20}$ However, the alloying element aluminum and rare-earths remain at the implant's site and accumulate by up to 60 wt.\%. In earlier studies, even after 3.5 years, parts of an LAE442 implant were observed in a rabbit's tibia. ${ }^{13}$ So far, no toxic reactions have been observed. However, such a long dwell time of the degradation products at the implant's location might have, in the long run, a negative impact. ${ }^{13}$ If the alloy consists only of elements that are also contained in the body at higher amounts, the degradation layer should be more easily resorbable by the body. $\mathrm{Ca}$ and $\mathrm{Zn}$ are trace elements in bone and occur in higher amounts than $\mathrm{Al}$, not to mention rare earths. Consequently, $\mathrm{Zn}$ and $\mathrm{Ca}$ are interesting alloying elements and thus are also being investigated for use in biodegradable $\mathrm{Mg}$ alloys. $\mathrm{Gu}$ et al. found that $\mathrm{Mg}-\mathrm{Zn}-\mathrm{Ca}$ alloys increased the bone formation and the density of the bone upon implantation. ${ }^{32}$ In the present study, a degradation rate between those of LAE442 and Mg-La2 could be realized using the ZX61 alloy. Furthermore, only a thin corrosion layer was observed in the in vitro experiments. A fast resorbing degradation layer is clearly less protective for the metallic substrate, i.e., will result in a faster overall degradation rate, but at the same time it also prevents the elements from accumulating at the implant's location. If a lower corrosion rate is desired, different coatings can be used, as demonstrated in the present study for the LAE442 and Mg-La2 implants. Similarly, Dou et al. used a MAO coating on Mg-Zn-Ca alloys and reduced the weight loss after 30 days by $70 \%$ compared with the uncoated alloy. Ding et al. also investigated the in vitro behavior of $\mathrm{Mg}-\mathrm{Zn}-\mathrm{Ca}$ alloys. According to their results, the corrosion potential, current density, and weight loss were further reduced by using two coating layers, viz. a MAO base with HA on top. ${ }^{33}$ Additional alloying elements which are also found as trace elements in the body, such as strontium (Sr), can be used to 
decrease the corrosion rate by building a protective Sr-HA layer. ${ }^{34,35}$ In vivo measurements also showed that $\mathrm{Mg}-\mathrm{Zn}-\mathrm{Ca}$ alloys did not have toxic effects on the metabolism. ${ }^{36}$ From the results obtained in the present study and data reported in literature, it is clear that Mg-based alloys can be tailored by variation of the chemical composition and the use of appropriate coatings to achieve a broad range of degradation rates. However, the long-term effects of accumulation of foreign elements in the degradation layer remain unclear. Although a long-term in vivo study of the degradation behavior of open-pored ZX61 scaffolds is not yet available, it appears that the less stable degradation layer observed for the ZX61 alloy in combination with a slowly degrading coating is a promising means to address this issue.

\section{CONCLUSION}

The degradation behavior of three different $\mathrm{Mg}$ based alloys intended for use in open-pored bioresorbable bone implants was analyzed by both in vitro and in vivo experiments. The main results can be summarized as follows:

1. The alloys' behavior in the in vitro and in vivo experiments was substantially different. Still, in vitro tests are a useful means to compare different alloys with respect to the degradation behavior.

2. The degradation rate could be successfully decreased by coating the implants with $\mathrm{MgF}_{2}$, $\mathrm{MgF}_{2}+$ polylactide acid (PLA), and $\mathrm{MgF}_{2}+$ calcium phosphate, of which the combination $\mathrm{MgF}_{2}+$ PLA was the most effective. This ranking of the coatings with respect to improved corrosion resistance was independent of the substrate employed.

3. From a mechanical viewpoint, the loading situation in the scaffold designed is not challenging, and thus all three alloys would be adequate in this respect. However, Mg-La2 does degrade too fast for such an application even if an $\mathrm{MgF}_{2}+$ PLA coating is applied.

4. The degradation rate of LAE442 was, compared with Mg-La2, much lower in both the in vitro as well as in vivo experiments, and could be used for the envisaged application.

5. However, in LAE442 implants, the aluminum and rare earths alloying elements were found to remain and accumulate to high concentrations at the implantation site. Although no negative clinical complications are known to date, the long-term effects of such accumulation of alloying elements remain unclear at the moment.

6. The ZX61 magnesium alloy is free of aluminum and rare earths. As intended, it exhibited a slow degradation rate, while the thin degradation layer indicates good resorption of the implant after its functional phase.

\section{ACKNOWLEDGEMENTS}

Open Access funding provided by Projekt DEAL. Financial supported by the German Research Foundation (DFG) within the project "Interfacial effects and ingrowing behavior of magnesium-based foams as bioresorbable bone substitute material" (Grant No. 271761343) is gratefully acknowledged. The Xradia 520 Versa used in this study was also funded by the DFG (Grant No. 316923185).

\section{OPEN ACCESS}

This article is licensed under a Creative Commons Attribution 4.0 International License, which permits use, sharing, adaptation, distribution and reproduction in any medium or format, as long as you give appropriate credit to the original author(s) and the source, provide a link to the Creative Commons licence, and indicate if changes were made. The images or other third party material in this article are included in the article's Creative Commons licence, unless indicated otherwise in a credit line to the material. If material is not included in the article's Creative Commons licence and your intended use is not permitted by statutory regulation or exceeds the permitted use, you will need to obtain permission directly from the copyright holder. To view a copy of this licence, visit $h$ ttp://creativecommons.org/licenses/by/4.0/.

\section{REFERENCES}

1. J.O. Hollinger and J.C. Kleinschmidt, The critical size defect as an experimental model to test bone repair materials. $J$. Craniofac. Surg. 1, 60 (1990). https://doi.org/10.1097/00001 665-199001000-00011.

2. P.C. Missiuna, H.S. Gandhi, F. Farrokhyar, B.E. Harnett, E.M.G. Dore, and B. Roberts, Anatomically safe and minimally invasive transcrestal technique for procurement of autogenous cancellous bone graft from the mid-iliac crest. Can. J. Surg. 54, 327 (2011). https://doi.org/10.1503/cjs.028 010 .

3. A.J. Salgado, O.P. Coutinho, and R.L. Reis, Bone tissue engineering: state of the art and future trends. Macromol. Biosci. 4, 743 (2004). https://doi.org/10.1002/mabi.20040002 6.

4. M. Bongio, J.J.J.P. van den Beucken, S.C.G. Leeuwenburgh, and J.A. Jansen, Development of bone substitute materials: from 'biocompatible' to 'instructive'. J. Mater. Chem. 20, 8747 (2010). https://doi.org/10.1039/C0JM00795A.

5. T. Douillard, J. Chevalier, A. Descamps-Mandine, I. Warner, Y. Galais, P. Whitaker, J.J. Wu, and Q.Q. Wang, Comparative ageing behaviour of commercial, unworn and worn 3Y-TZP and zirconia-toughened alumina hip joint heads. J. Eur. Ceram. Soc. 32, 1529 (2012). https://doi.org/ 10.1016/j.jeurceramsoc.2012.01.003.

6. J. Zhang, W. Liu, V. Schnitzler, F. Tancret, and J.-M. Bouler, Calcium phosphate cements for bone substitution: chemistry, handling and mechanical properties. Acta Biomater. 10, 1035 (2014). https://doi.org/10.1016/j.actbio.2013. 11.001.

7. W. Bonfield, Composites for bone replacement. J. Biomed. Eng. 10, 522 (1988). https://doi.org/10.1016/0141-5425(88)9 0110-0.

8. D.R. Sumner, Long-term implant fixation and stressshielding in total hip replacement. J. Biomech. 48, 797 (2015). https://doi.org/10.1016/j.jbiomech.2014.12.021. 
9. J. Malekani, B. Schmutz, Y. Gu, M. Schuetz, P. Yarlagadda in Proceedings of the 2nd Annual International Conference on Materials Science, Metal \& Manufacturing. Biomaterials in orthopedic bone plates a review (2011), pp. 71-77.

10. X.-N. Gu and Y.-F. Zheng, A review on magnesium alloys as biodegradable materials. Front. Mater. Sci. China 4, 111 (2010). https://doi.org/10.1007/s11706-010-0024-1.

11. S. Julmi, A.-K. Krüger, A.-C. Waselau, A. Meyer-Lindenberg, P. Wriggers, C. Klose, and H.J. Maier, Processing and coating of open-pored absorbable magnesium-based bone implants. Mater. Sci. Eng. C Mater. Biol. Appl. 98, 1073 (2019). https://doi.org/10.1016/j.msec.2018.12.125.

12. Y. Xin, T. Hu, and P.K. Chu, In vitro studies of biomedical magnesium alloys in a simulated physiological environment: a review. Acta Biomater. 7, 1452 (2011). https://doi.org/10. 1016/j.actbio.2010.12.004

13. N. Angrisani, J. Reifenrath, F. Zimmermann, R. Eifler, A. Meyer-Lindenberg, K. Vano-Herrera, and C. Vogt, Biocompatibility and degradation of LAE442-based magnesium alloys after implantation of up to 3.5 years in a rabbit model. Acta Biomater. 44, 355 (2016). https://doi.org/10.1016/j.actb io.2016.08.002.

14. E. Willbold, X. Gu, D. Albert, K. Kalla, K. Bobe, M. Brauneis, C. Janning, J. Nellesen, W. Czayka, W. Tillmann, Y. Zheng, and F. Witte, Effect of the addition of low rare earth elements (lanthanum, neodymium, cerium) on the biodegradation and biocompatibility of magnesium. Acta Biomater. 11, 554 (2015). https://doi.org/10.1016/j.actbio.20 14.09.041.

15. J.R. Lieberman and G.E. Friedlaender, Bone Regeneration and Repair: Biology and Clinical Applications (Totowa: Humana, 2005).

16. S. Julmi, C. Klose, A.-K. Krüger, P. Wriggers, H.J. Maier, in T.M.M.\&M.S.TMS (Ed.), TMS 2017 146th Annual Meeting \& Exhibition Supplemental Proceedings. Development of sponge structure and casting conditions for absorbable magnesium bone implants (Springer, Cham, 2017), pp. 307-317.

17. J.-M. Seitz, K. Collier, E. Wulf, D. Bormann, N. Angrisani, A. Meyer-Lindenberg, and F.-W. Bach, The effect of different sterilization methods on the mechanical strength of magnesium based implant materials. Adv. Eng. Mater. 13, 1146 (2011). https://doi.org/10.1002/adem.201100074.

18. A. Weizbauer, J.-M. Seitz, P. Werle, J. Hegermann, E. Willbold, R. Eifler, H. Windhagen, J. Reifenrath, and H. Waizy, Novel magnesium alloy Mg-2La caused no cytotoxic effects on cells in physiological conditions. Mater. Sci. Eng. C Mater. Biol. Appl. 41, 267 (2014). https://doi.org/10.1016/j. msec.2014.04.063.

19. A. Oyane, H.-M. Kim, T. Furuya, T. Kokubo, T. Miyazaki, and T. Nakamura, Preparation and assessment of revised simulated body fluids. J. Biomed. Mater. Res. A 65, 188 (2003). https://doi.org/10.1002/jbm.a.10482.

20. N. Kleer, S. Julmi, A.-K. Gartzke, J. Augustin, F. Feichtner, A.-C. Waselau, C. Klose, H.J. Maier, P. Wriggers, and A. Meyer-Lindenberg, Comparison of degradation behaviour and osseointegration of the two magnesium scaffolds, LAE442 and La2, in vivo. Materialia 8, 100436 (2019). h ttps://doi.org/10.1016/j.mtla.2019.100436.

21. A. Zioła-Frankowska, Ł. Kubaszewski, M. Dabrowski, A. Kowalski, P. Rogala, W. Strzyżewski, W. Łabędź, R. Uklejewski, K. Novotny, V. Kanicky, and M. Frankowski, The content of the 14 metals in cancellous and cortical bone of the hip joint affected by osteoarthritis. Biomed. Res. Int. 2015, 815648 (2015). https://doi.org/10.1155/2015/815648.

22. H.R. Bakhsheshi-Rad, E. Hamzah, A. Fereidouni-Lotfabadi, M. Daroonparvar, M.A.M. Yajid, M. Mezbahul-Islam, M. Kasiri-Asgarani, and M. Medraj, Microstructure and biocorrosion behavior of $\mathrm{Mg}-\mathrm{Zn}$ and $\mathrm{Mg}-\mathrm{Zn}-\mathrm{Ca}$ alloys for biomedical applications. Mater. Corros. 65, 1178 (2014). h ttps://doi.org/10.1002/maco.201307588.
23. J. Reifenrath, D. Gottschalk, N. Angrisani, S. Besdo, and A. Meyer-Lindenberg, Axial forces and bending moments in the loaded rabbit tibia in vivo. Acta Vet. Scand. 54, 21 (2012). https://doi.org/10.1186/1751-0147-54-21.

24. Volkswagen AG, 3D-Druck weltweit (2017). www.volkswage nag.com. Accessed 20 June 2019.

25. K.Y. Chiu, M.H. Wong, F.T. Cheng, and H.C. Man, Characterization and corrosion studies of fluoride conversion coating on degradable $\mathrm{Mg}$ implants. Surf. Coat. Technol. 202, 590 (2007). https://doi.org/10.1016/j.surfcoat.2007.06.0 35.

26. M. Thomann, C. Krause, N. Angrisani, D. Bormann, T. Hassel, H. Windhagen, and A. Meyer-Lindenberg, Influence of a magnesium-fluoride coating of magnesium-based implants $(\mathrm{MgCa} 0.8)$ on degradation in a rabbit model. $J$. Biomed. Mater. Res. A 93, 1609 (2010). https://doi.org/10. 1002/jbm.a.32639.

27. L. Wolters, S. Besdo, N. Angrisani, P. Wriggers, B. Hering, J.-M. Seitz, and J. Reifenrath, Degradation behaviour of LAE442-based plate-screw-systems in an in vitro bone model. Mater. Sci. Eng. C Mater. Biol. Appl. 49, 305 (2015). https://doi.org/10.1016/j.msec.2015.01.019.

28. R.-C. Zeng, W.-C. Qi, Y.-W. Song, Q.-K. He, H.-Z. Cui, and E.-H. Han, In vitro degradation of MAO/PLA coating on Mg1.21Li-1.12Ca-10Y alloy. Front. Mater. Sci. 8, 343 (2014). h ttps://doi.org/10.1007/s11706-014-0264-6.

29. A. Abdal-hay, N.A.M. Barakat, and J.K. Lim, Hydroxyapatite-doped poly(lactic acid) porous film coating for enhanced bioactivity and corrosion behavior of AZ31 Mg alloy for orthopedic applications. Ceram. Int. 39, 183 (2013). h ttps://doi.org/10.1016/j.ceramint.2012.06.008.

30. S. Tanodekaew, S. Channasanon, P. Kaewkong, and P. Uppanan, PLA-HA scaffolds: preparation and bioactivity. Proc. Eng. 59, 144 (2013). https://doi.org/10.1016/j.proeng. 2013.05.104.

31. D. da Silva, M. Kaduri, M. Poley, O. Adir, N. Krinsky, J. Shainsky-Roitman, and A. Schroeder, Biocompatibility, biodegradation and excretion of polylactic acid (PLA) in medical implants and theranostic systems. Chem. Eng. J. 340, 9 (2018). https://doi.org/10.1016/j.cej.2018.01.010.

32. X. Gu, F. Wang, X. Xie, M. Zheng, P. Li, Y. Zheng, L. Qin, and $\mathrm{Y}$. Fan, In vitro and in vivo studies on as-extruded $\mathrm{Mg}$ 5.25wt.\%Zn-0.6wt.\%Ca alloy as biodegradable metal. Sci. China Mater. 61, 619 (2018). https://doi.org/10.1007/s40843017-9205-x.

33. H.-Y. Ding, H. Li, G.-Q. Wang, T. Liu, and G.-H. Zhou, Biocorrosion behavior of ceramic coatings containing hydroxyapatite on Mg-Zn-Ca magnesium alloy. Appl. Sci. 8, 569 (2018). https://doi.org/10.3390/app8040569.

34. M. Bornapour, H. Mahjoubi, H. Vali, D. Shum-Tim, M. Cerruti, and M. Pekguleryuz, Surface characterization, in vitro and in vivo biocompatibility of $\mathrm{Mg}-0.3 \mathrm{Sr}-0.3 \mathrm{Ca}$ for temporary cardiovascular implant. Mater. Sci. Eng. C Mater. Biol. Appl. 67, 72 (2016). https://doi.org/10.1016/j.msec. 2016.04.108.

35. X.N. Gu, X.H. Xie, N. Li, Y.F. Zheng, and L. Qin, In vitro and in vivo studies on a Mg-Sr binary alloy system developed as a new kind of biodegradable metal. Acta Biomater. 8, 2360 (2012). https://doi.org/10.1016/j.actbio.2012.02.018.

36. G. Bao, Q. Fan, D. Ge, M. Sun, H. Guo, D. Xia, Y. Liu, J. Liu, $\mathrm{S}$. Wu, B. He, and Y. Zheng, In vitro and in vivo studies on magnesium alloys to evaluate the feasibility of their use in obstetrics and gynecology. Acta Biomater. 97, 623 (2019). h ttps://doi.org/10.1016/j.actbio.2019.08.001.

Publisher's Note Springer Nature remains neutral with regard to jurisdictional claims in published maps and institutional affiliations. 